\title{
Science ouverte, bibliodiversité et qualité éditoriale: l'exemple de la pépinière de revues en accès ouvert de Lyon 3
}

\author{
Développée au sein des bibliothèques universitaires de Lyon 3 , la pépinière de revues défend la \\ bibliodiversité et des pratiques éditoriales de qualité en matière d'open access.
}

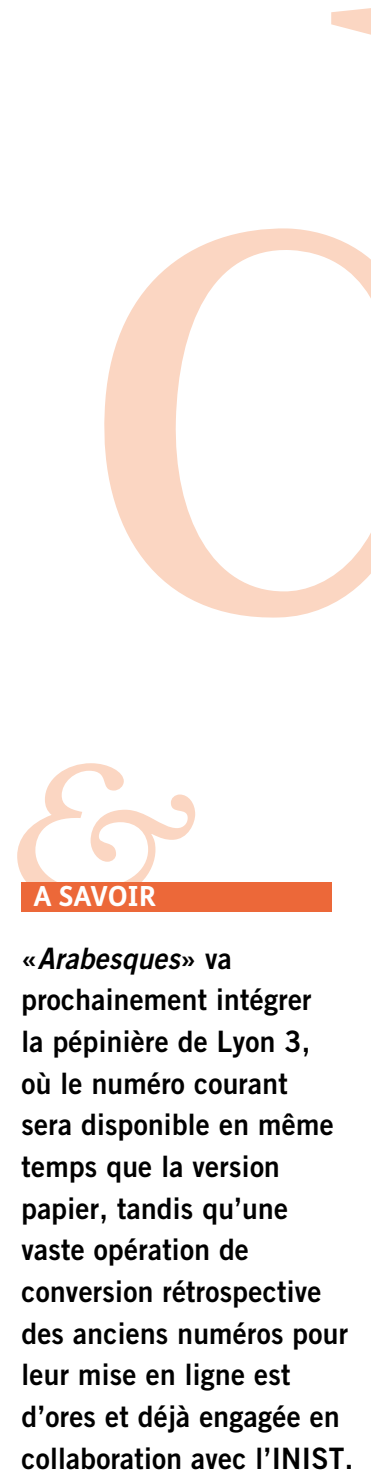

[1] Données fournies par Odile Contat, InSHS (Institut national des sciences humaines et sociales, CNRS).

[2] revues.univ-Iyon3.fr

[3] Antérieurement Revues.org : www.openedition.org
Depuis longtemps, le constat a été fait que la concentration sans précédent que connaît le monde de l'édition académique a considérablement affaibli et appauvri l'écosystème éditorial, notamment en sciences, techniques et médecine (STM). La situation est différente en sciences humaines et sociales (SHS), en particulier en France où les 2000 revues scientifiques produites dans ces disciplines sont encore publiées par une multitude de structures aux statuts très divers : éditeurs commerciaux ou universitaires, associations, laboratoires, etc.

L'écosystème est donc encore assez diversifié, mais il est en pleine évolution, notamment du fait du passage massif au numérique : en 2008 , $55 \%$ des revues françaises de SHS étaient encore uniquement au format papier, contre seulement 10 à $15 \%$ aujourd'hui ${ }^{1}$. Cette évolution très rapide, qui peut déstabiliser des équilibres antérieurs déjà fragiles, doit être accompagnée si l'on veut favoriser l'émergence d' " un environnement éditorial [...] obéissant aux principes d'un accès ouvert et éthique» que le Plan national pour la Science ouverte, ici cité, appelle de ses vœux.

\section{NAISSANCE ET DÉVELOPPEMENT DE LA PÉPINIÈRE}

C'est dans cet esprit que les BU de Lyon 3 ont créé, à la fin de l'année 2016, un portail de revues scientifiques en accès ouvert². Ce projet est né, en 2015, au sein du Service d'appui documentaire à la recherche qui venait de voir le jour. Ce service avait progressivement pris conscience que les revues portées par les unités de recherche de cette université, qui étaient au nombre de 13 pour 18 laboratoires, rencontraient de réelles difficultés : en 2016, huit d'entre elles étaient encore uniquement au format papier et souffraient d'une très faible visibilité ; d'autres, passées au numérique, s'appuyaient sur des solutions techniques peu adaptées à la diffusion de publications académiques. Seules deux revues avaient rejoint OpenEdition et disposaient, de ce fait, d'un cadre éditorial professionnalisé. Les autres étaient portées par des chercheurs peu acculturés aux questions éditoriales et ne disposant pas d'interlocuteurs au sein de l'université pour faire évoluer leurs revues vers le numérique dans des conditions satisfaisantes.
La création d'une pépinière de revues en accès ouvert impliquait une évolution importante dans le positionnement des BU au sein de l'université Lyon 3, car l'édition n'entre pas dans le champ de compétences traditionnel des bibliothèques. Mais cette évolution était déjà en germe dans l'activité développée au sein du service qui venait de naître: en prenant en charge l'administration du portail HAL de l'université ou la diffusion numérique des thèses et des mémoires de master recherche, la BU assurait, de plus en plus, la diffusion en accès ouvert d'une production scientifique numérique produite au sein de l'établissement et restée jusque-là relativement confidentielle. La création d'une pépinière de revues n'a constitué que le point d'aboutissement d'un processus, largement favorisé par le développement de la science ouverte, où la BU est conduite à s'impliquer dans l'ensemble du processus de production, de diffusion, de valorisation et de conservation du savoir scientifique produit par son institution.

Cette évolution a un coût, car elle oblige les bibliothécaires à s'aventurer sur des territoires mal connus, les métiers de l'édition ou ceux des archives pour les SCD impliqués dans le soutien à l'Open data. Elle impose donc d'acquérir des compétences nouvelles, mais elle permet en retour de tisser des liens différents et souvent plus étroits avec les chercheurs, comme a pu l'expérimenter l'équipe qui a mis en place la pépinière de revues.

\section{ACCOMPAGNER LES JEUNES POUSSES}

Le terme de " pépinière " n'est pas anodin. Dès l'origine du projet, l'objectif affiché était de permettre aux revues hébergées sur le portail de Lyon 3 de rejoindre, à terme, OpenEdition ${ }^{3}$. II s'agissait donc de créer des revues numériques pour les laboratoires, mais aussi d'offrir un accompagnement aux équipes éditoriales afin de leur permettre de monter en compétence, d'améliorer la qualité de leurs publications puis de candidater pour rejoindre cette plateforme disposant d'un catalogue de 500 revues, d'une visibilité internationale, mais relativement sélective. Cela a conduit l'équipe à s'appuyer sur les outils développés par OpenEdition, notamment le logiciel libre Lodel, pour créer l'infrastructure du portail et permettre ainsi aux porteurs des revues de passer 
d'un environnement à l'autre sans changement majeur dans leurs pratiques.

Le portail des revues de Lyon 3 est donc pensé comme un espace de diffusion provisoire, sauf pour certaines publications n'ayant pas vocation à rejoindre OpenEdition. II constitue une étape dans la vie de la revue pendant laquelle l'équipe de la pépinière apporte son aide pour former les secrétaires de publication, préciser le cadre juridique de la revue, améliorer les processus éditoriaux... Le travail mené avec les responsables des Cahiers Jean Moulin ${ }^{4}$, de septembre 2016 à avril 2017, a permis de définir les éléments de base de l'offre de services proposée par les BU de Lyon 3 (gratuité des services, insistance sur l'accompagnement de proximité, accent mis sur l'open access). La collaboration menée avec les cinq revues qui ont ensuite rejoint la pépinière a permis de l'affiner et de l'enrichir.

\section{BIBLIODIVERSITÉ ET BONNES PRATIQUES ÉDITORIALES}

Le portail de Lyon 3 accueille aujourd'hui 6 revues en accès ouvert et offrira, en juin 2019, un hébergement à l'ensemble des publications des laboratoires de Lyon 3 qui rencontraient, en 2016, des problèmes de diffusion. Le rythme d'intégration tend à s'accélérer puisque 6 revues rejoindront, en 2019, la pépinière, qui élargit aussi son périmètre d'intervention en entamant un travail avec des équipes éditoriales ne dépendant pas de Lyon 3.

Cette dernière évolution est significative des difficultés que rencontrent, en France, les responsables éditoriaux pour trouver un espace de diffusion en accès ouvert pour leurs publications. Une plateforme comme OpenEdition reste encore un exemple trop isolé et, face au développement d'un accès ouvert reposant sur des frais de publication demandés aux auteurs, il devient urgent de soutenir une édition publique reposant sur le financement en amont de plateformes, comme le modèle " diamant » tente de le promouvoir. L'émergence depuis quelques années de pépinières de revues portées par des BU mais aussi par des Maisons des sciences de I'homme ${ }^{5}$ constitue un embryon de réponse allant dans ce sens. Ces pépinières peuvent apporter un accompagnement de proximité, répondre aux revues dans des délais relativement courts, voire se lancer dans des formes d'expérimentation éditoriale. Elles participent à l'émergence d'un écosystème plus riche, doté d'opérateurs de tailles différentes favorisant les jeux d'échelle.

Mais, pour véritablement défendre une bibliodiversité vertueuse, ces pépinières doivent monter en compétence et agir en concertation avec les grands acteurs de l'édition scientifique publique. C'est dans cette logique que le portail de revues de Lyon 3 est devenu, en juillet 2018, la première pépinière officielle d'OpenEdition ${ }^{6}$ et que l'équipe

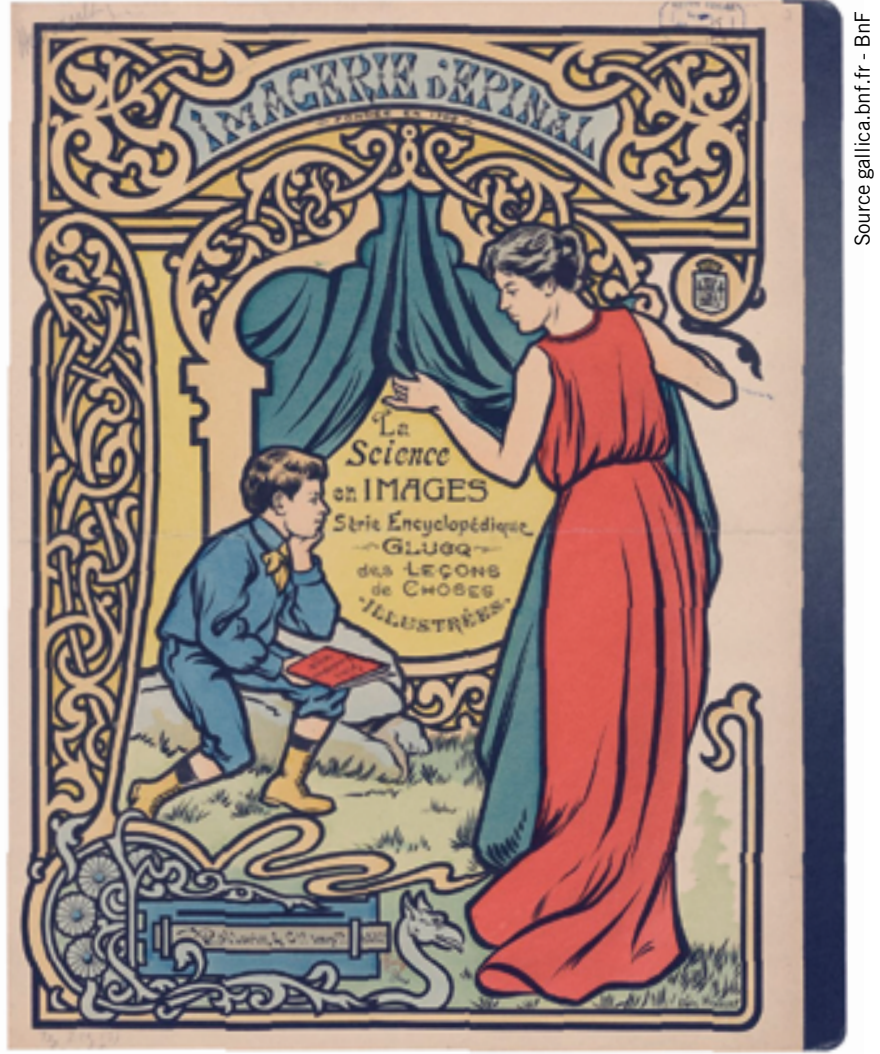

qui la porte a organisé, le 23 novembre 2018, avec la MSH de Dijon, une journée de travail réunissant onze pépinières de revues venues de France et de Belgique, des représentants des infrastructures de recherche Métopes ${ }^{7}$ et OpenEdition, de Médici (le réseau des professionnels de l'édition scientifique publique) de l'ADBU et de l'InSHS. De cette réunion est né Repères (Réseau de pépinières de revues scientifiques en accès ouvert) dont l'un des objectifs est de favoriser, au sein de ce réseau mais aussi au-delà, l'adoption de bonnes pratiques éditoriales. L'enjeu est important, car le développement d'une édition en accès ouvert doit encourager la réintroduction de formes de régulation et de transparence dans les pratiques éditoriales, et s'appuyer sur des exigences éthiques fortes, comme tentent de les promouvoir Latindex ${ }^{8}$ ou le DOAJ ${ }^{9}$, deux portails de référencement de revues en accès ouvert qui ont établi des critères de qualité éditoriale qui constituent aujourd'hui des standards internationaux servant de base au travail de Repères.

Un précurseur de la défense de l'environnement avait pour slogan : "Penser global, agir local » ${ }^{\mathbf{1 0}}$. La défense de la bibliodiversité nécessite aussi cette double approche que tentent, à leur échelle, de mettre en œuvre les BU de Lyon 3.

Jean-Luc de Ochandiano Responsable des Services documentaires aux chercheurs Bibliothèques universitaires Jean Moulin Lyon 3 jean-luc.de-ochandiano@univ-lyon3.fr

[7] www.metopes.fr
- La Science en images. Série encyclopédique Glucq des leçons de choses illustrées 1906 [couv. ill. par] Jules Hénault

[4] revues.univ-lyon3.fr/cjm [5] Voir en particulier la plateforme de la MSH de Dijon, Preo : preo.u-bourgogne.fr/portail [6] leo.hypotheses.org/14490

[8] www.latindex.org/latindex/ revistaselec

[9] Directory of open access Journals : doaj.org/fr/ editionsavante

[10] René Dubos lors du premier sommet sur l'environnement en 1972. 